\title{
NEW APPLICATIONS OF RADIO HOLOGRAPHY FOR MM OBSERVATIONS WITH THE RATAN-600 RADIO TELESCOPE
}

\section{VLADIMIR KHAIKIN}

The Special Astrophysical Oboervatory of Russian Academy of Sciences, N.Arthyz, 357147 , Karachai-Cherkessia, Russia

Tx:123244 ZENIT SU,FAX:(7.812)s16-17.01,emailivk Osao.stauropol.eu

\section{ABSTRACT}

In the paper we describe the results of active holography RATAN-600 ourface testing including the measurements of a single element profile and topography. The RATAN-600 beam pattern at $3.2 \mathrm{~mm}$ after an antenna correction and its dynamica during daytime are preaented.

\section{INTRODUCTION}

The RATAN-600 has been planned and built as an instrument enabling os to reach maximum resolution for a reflector radio telescope in the wide wave range from 30 to $1 \mathrm{~cm}$ (Korolkov, Parijskij, 1979). The improvement of the radio telescope reflecting ourface and the development of holography surface testing have provided as with the possibility to work in MM wave range of a very big reflector and in future - of a big array(Parijokij et al.,1892). New radio holography applications help us to achieve the above mentioned goals.

\section{THE HOLOGRAPHY TECHNIQUE AND EQUIPMENT}

Figure 1: A scheme of holography measurements at the RATAN-600

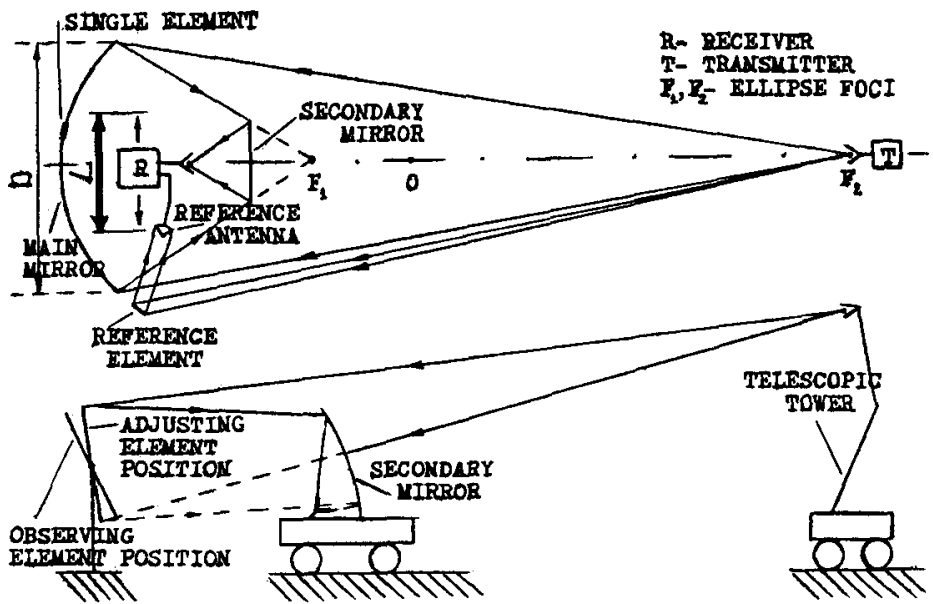


The main advantage of the method we ase is radio holography measurements in the mode which is maximaly close to the process of radio astronomical observations (Khaikin,1891). Fig.1 gives a scheme of holography measurements at the RATAN-600 with the noe of a remote ground based signal source.

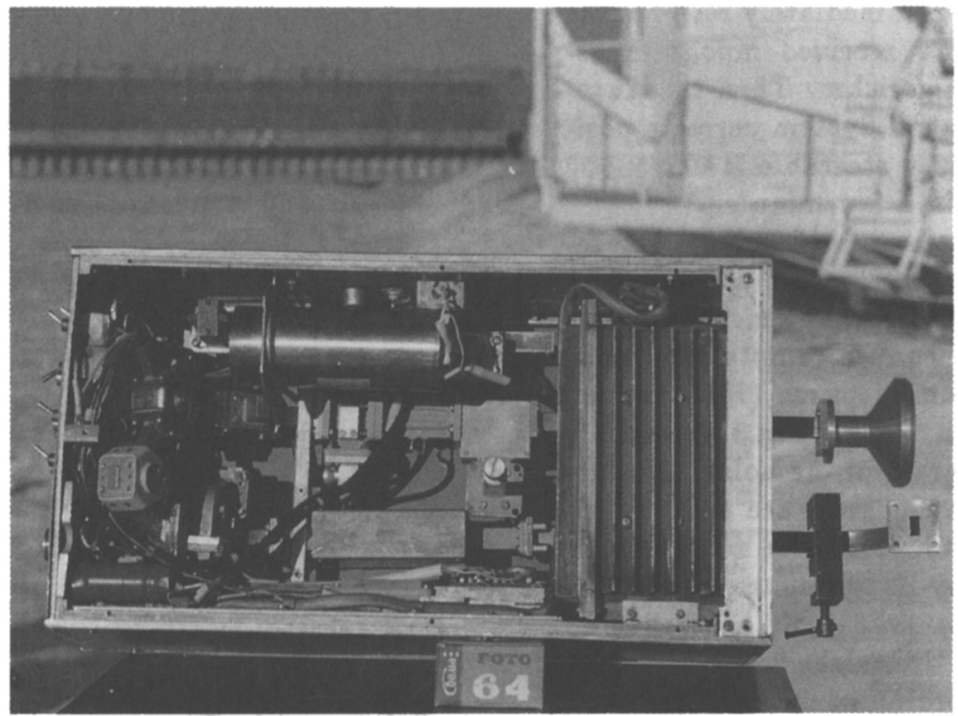

Figure 2: The two-channel holography receiver at $22 \mathrm{GHz}$

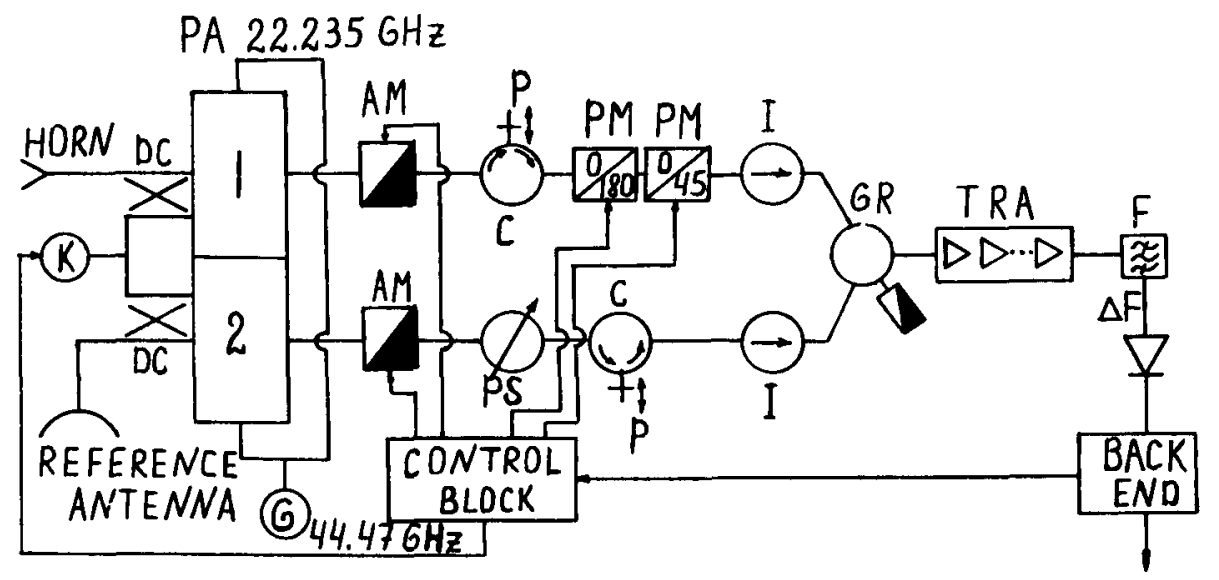

Figure 3: A block diagram of the holography receiver. PA - twochannel parametric amplifter with common pumping, PS - linear phase shifter, PM - phase modulator, GR - gibride ring, TRA - FET amplifler, AM - amplitude modulator, G - pumping, $K$ - calibration 
A radio hologram is recorded while the platform with the holography receiver is moving along the focal line of the Secondary mirror. IBM AT 386 placed in the Laboratory building immediately restores the "ON-LINE" received hologram asing FFT algorithm. Then the Automatic Control System corrects positions of folly steerable RATAN-600 elements in a few minutes.

The two-channel holography receiver at $22.235 \mathrm{GHz}$ specially designed for holography measurements with the use of a remote ground or strong cosmic source ( $\mathrm{H}_{2} \mathrm{O}$ maser) is given in Fig.2. Fig.3 presents a block diagram of the holography receiver. A linear phase shift is applied in the receiver to avoid outp ut holography image distortions (Bennet et al., 1976). It is similar to the Leith-Upatnieks "off-axis" hologram recording in optics.

The characteristics of the receiver and phase devices used are given in (Khaikin, Yaremenko, 1991;

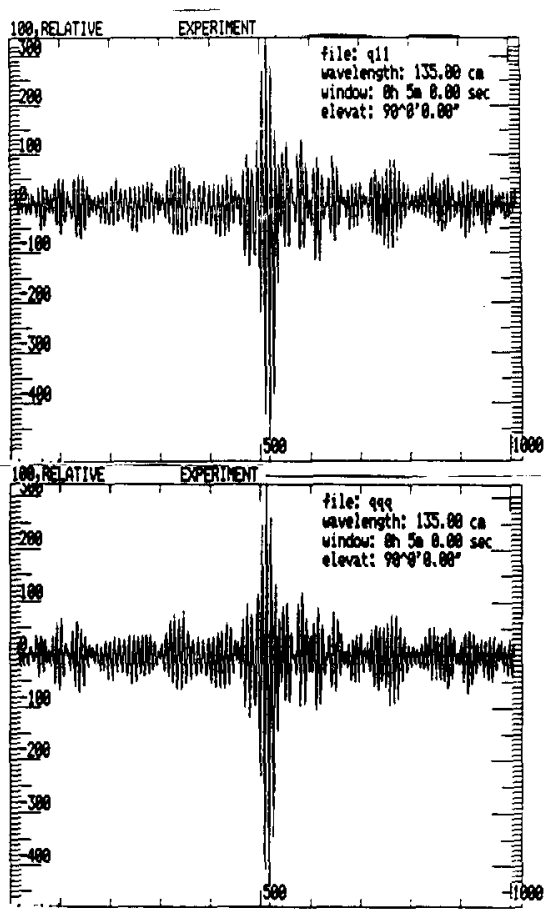

Figure 4: Two holograms recorded with 0.6 hour difference Romanov et all., 1981). High phase atability of the receiver front end (Dvoyan et al, 1981) guaranteeg good quality of holography recording. In Fig.4 two one-dimensional holograms recorded in the daytime with 0.5 hour difference demonstrate a high repeatability of holography recording.

\section{HOLOGRAPHY TESTING OF THE RATAN-600 SURFACE}

Fig.5. presents the results of holography measuremente of the RATAN-600 anrface with element numbers from 640 to 670 before and after the antenn a correction. The subatantial improvement of both the aperture phase(radial element positions) and amplitude (angle element positions) are demonstrated. As the reault r.m.s. accuracy of mutual elements alignment is improved from $\sigma=0.54 \mathrm{~mm}$ to $\sigma=0.083 \mathrm{~mm}$. Residual surface distortions correspond to the RATAN-600 Automatic Control System precision. The accuracy of single measurement performed by this method during the daytime is equal to $50 \mu \mathrm{m}$.

The complete cycle holography meagarements - antenna correction - holography measurements for radio telescope sector (225 element) can be performed in 15-20 min if the most important radial coordinate is corrected. To correct all the three coordinates several one-dimensional holograms with different angle antenna positions should be recorded and an antenna correction takes 30-35 min. 


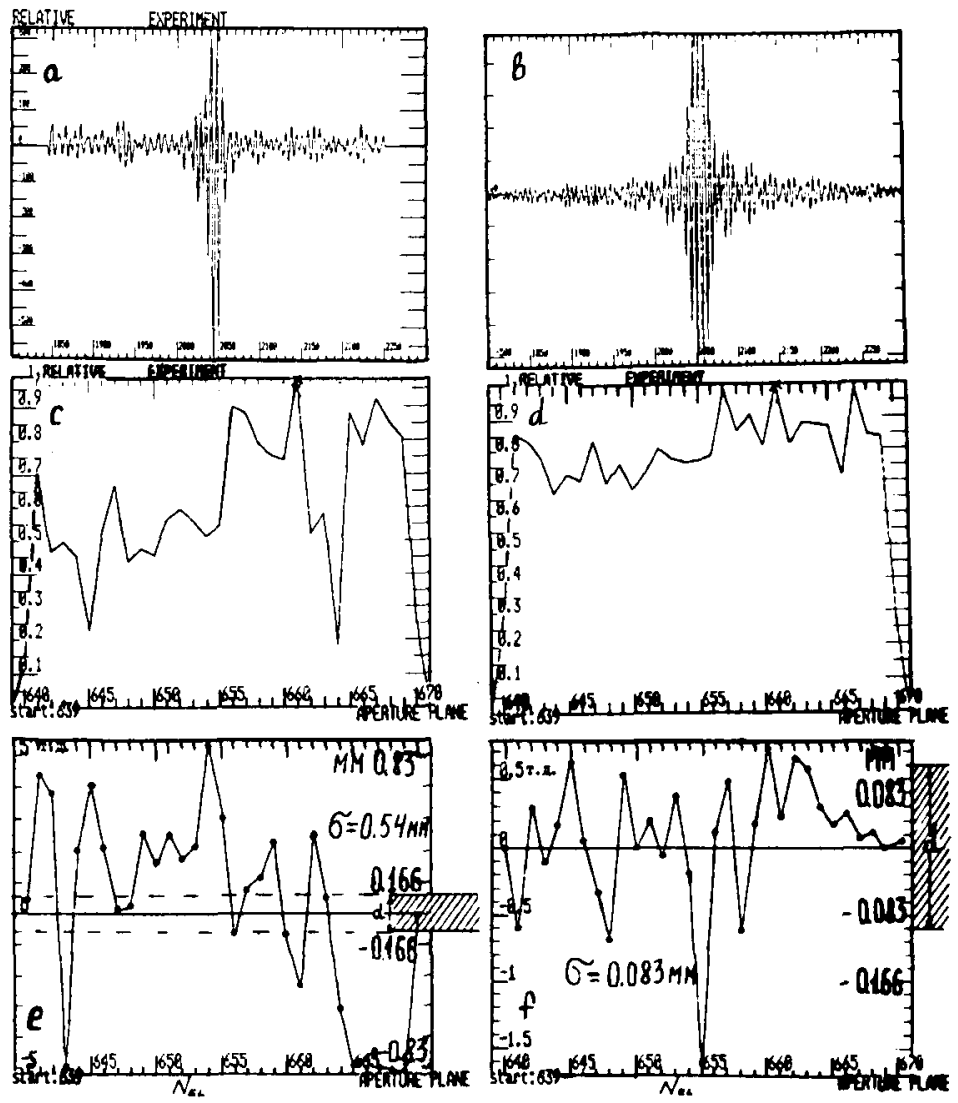

Figure 5: Holography testing and correction of the RATAN-600 surface. $N_{E L}$-element number, holograms $(a, b)$, aperture amplitude distributions (c,d) and radial element positions ( $e, f)$ before (left) and after(right) the antenna correction

\section{HOLOGRAPHY MEASUREMENTS OF A SINGLE RATAN-600 ELEM ENT}

In Fig.6. the holography measurements of surface in tegral profiles ( by vertical coordinate) for elements numbered 640 and 670 are given. The concavity(el.670) or the salience(el.640) of the restored integral profiles are the result of the difference between the real curvature radius $\left(R_{r}\right)$ of the given element and optimal one $\left(R_{o}\right)$. The calculation of $R_{o}$, which depends on the element position, focus position etc, allows ds to estimate $R_{\text {r }}$ for given element. For 640 and 670 elements occasional deviations of the ideal profile form are $\sigma=0.16 \mathrm{~mm}$ and $\sigma=0.11 \mathrm{~mm}$. The integral profile measurement takes only $5 \mathrm{~min}$ with repeatability better than $10 \mu \mathrm{m}$ and horizontal resolution of $10 \mathrm{~cm}$.

Fig.7 demonstrates repeatability of the method. The integral profile of 
668 element is restored here by 3 holograms recorded one by one (exp.12-14). The r.m.s. error of the difference between the profiles in experiments 12 and 13 (except trend) is less then $2 \mu \mathrm{m}$. The biggeat profile difference abort $8 \mu \mathrm{m}$ is between the 12-th and 13-th experiments (Fig. 8).

To do a surface map of an antenna element a two-dimenional hologram
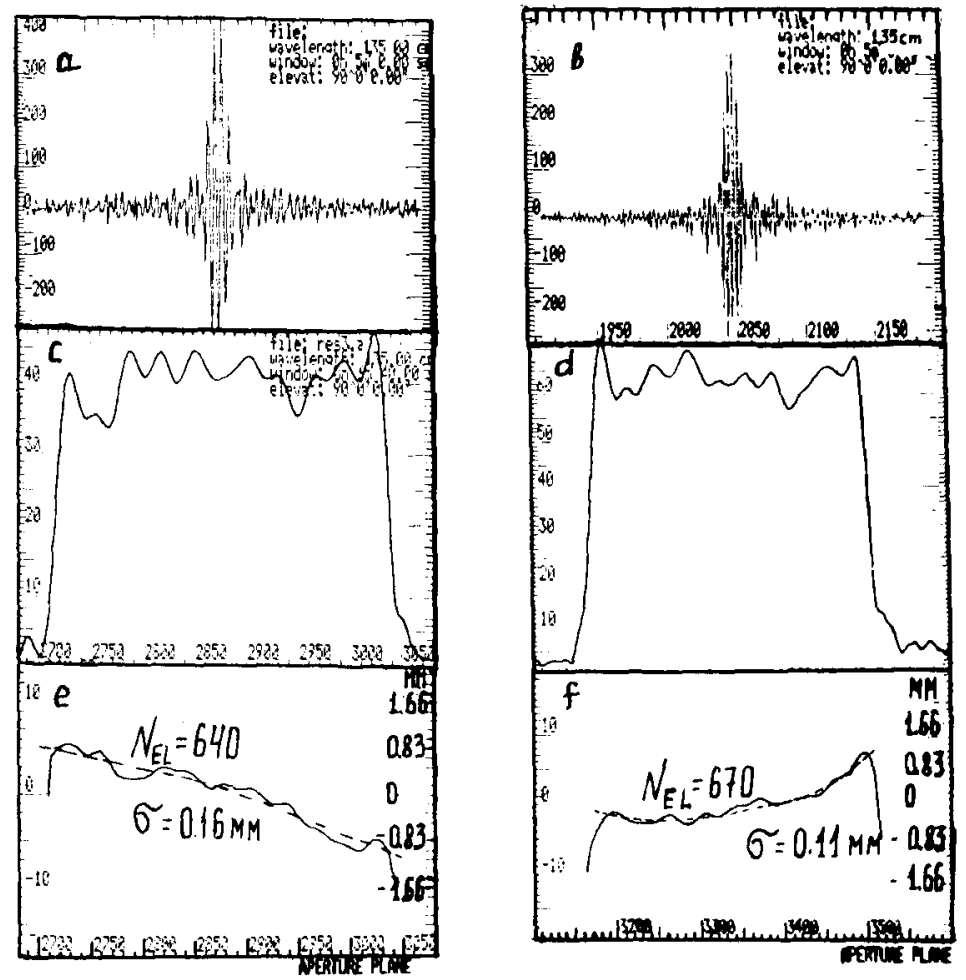

Figure 0: The holograms $(a, b)$, aperture fleld amplitudes(c,d) and surface integral proftles $(e, f)$ of 640 and 670 elements

should be recorded which at the required resolution of $20 \times 20 \mathrm{~cm}$ (the distance between the adjustment acrews at the panel onrface) takes 1.5 hours. The accaracy of a holography recording in this case is limited by amplitude and phase fuctuation of close to ground atmosphere level at the RATAN-600. $100 \mu \mathrm{m}$ accuracy of the restored surface map can be reached during the night time when the level of phase atmosphere fluctuations is usually less than $\sigma=3^{\circ}$.

To eatimate the influence of other factors distorting a two-dimenaional hologram and restored aperture field the far-field modeling of the reconstruction process was provided(Fig.9). The noise, limitations of recording region, non-linear phase shift etc. were taken account of. As the basic model we take a rectangular aperture whose small element has been phase shifted to $\lambda / 4$ (Fig.9 a)

Fig. 8 shows that the given field distribution in the apertare can be successfully restored using FFT even if the noise level is as high as $-40 \mathrm{~dB}(S / N$ 
Figure 7: The aperture amplitude distribution(left) and integral profle(right) of 860 element restored by 3 holograms

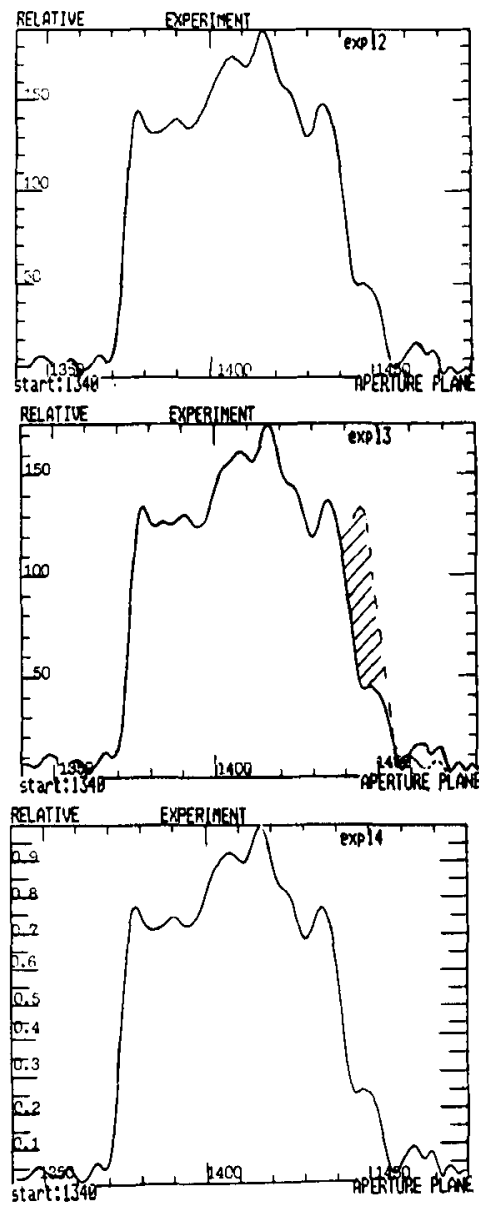

ratio in the hologram), bot catting of the hologram part, the noise and non-linearity of phase shift lead to extension of the output image size and the appearance of "higher" images(Fig. $8 \mathrm{i}-1$ ).

Fig. 10 presents 9 of 20 sections of the two-dimensional hologram recorded for one of the RATAN600 element. Daring the hologram recording a fnlly steerable element scans by the azimath coordinate with the angle shift ofter each scan.

Fig.11 showg the reanlts of the experimental hologram processing.
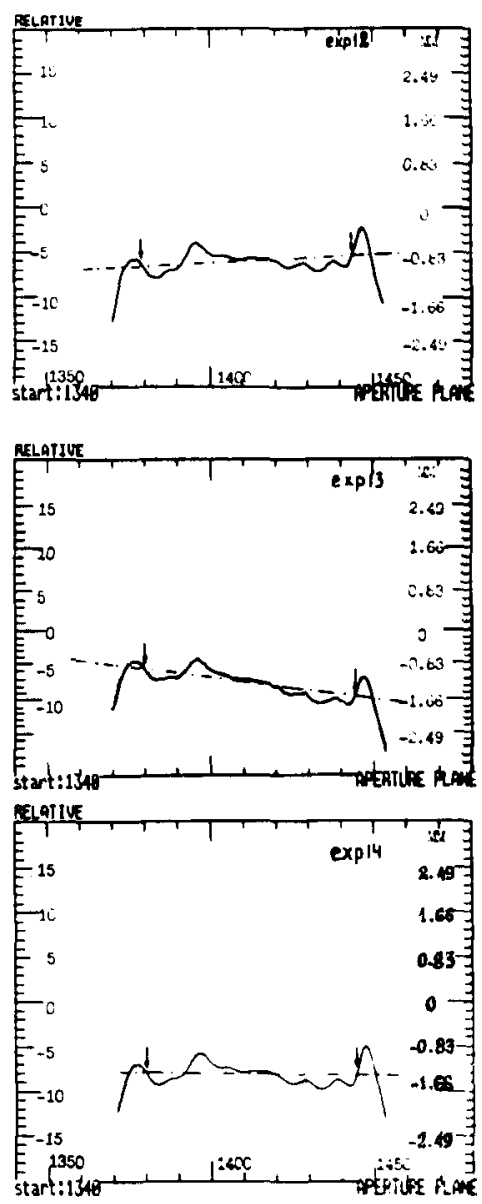

Figure 8: The difference between profles in 12 and 13 experiments

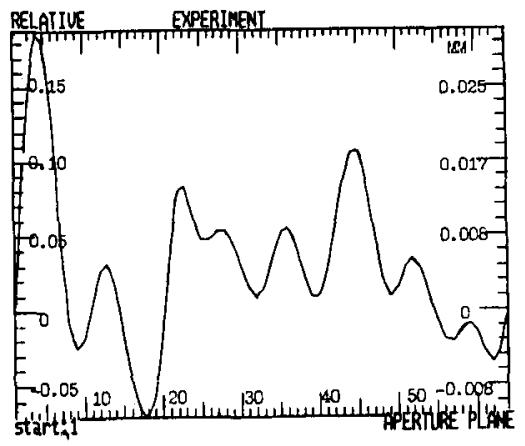


The primarily installed test plate of $85 \times 20 \mathrm{~cm}$ size can be clearly digtinguished at the restored aperture(Fig.11 g). Significent oscillations of the restored aperture amplitude in the real experiment (Fig.11 f) are induced by the near region position of the signal source(Fig 1). They can be excluded with the use of the Fresnel trangform algorithm. We consider the real resolation achieved in the ortput image to be equal $\delta y \times \delta x=40 \times 20 \mathrm{~cm}$. The extension of the hologram recording region could enable as to reach the required regolution of $20 \times 20 \mathrm{~cm}$.

\section{Figure 8:}

Modeling

of the aperture fleld reconstruction.

The given phase (a) and amplitude (b), restored real and imaginary aperture phase (left) and amplitude (right); $i, j$, $k, 1$ - images in the case of cutting of the hologram part. Noise level: $\mathbf{0}$ dB (c, d, i, j), $-40 \mathrm{~dB}$ $(\mathrm{e}, \mathrm{f}),-\mathbf{- s} \mathrm{dB}(\mathrm{B}, \mathrm{h}$, k, l)
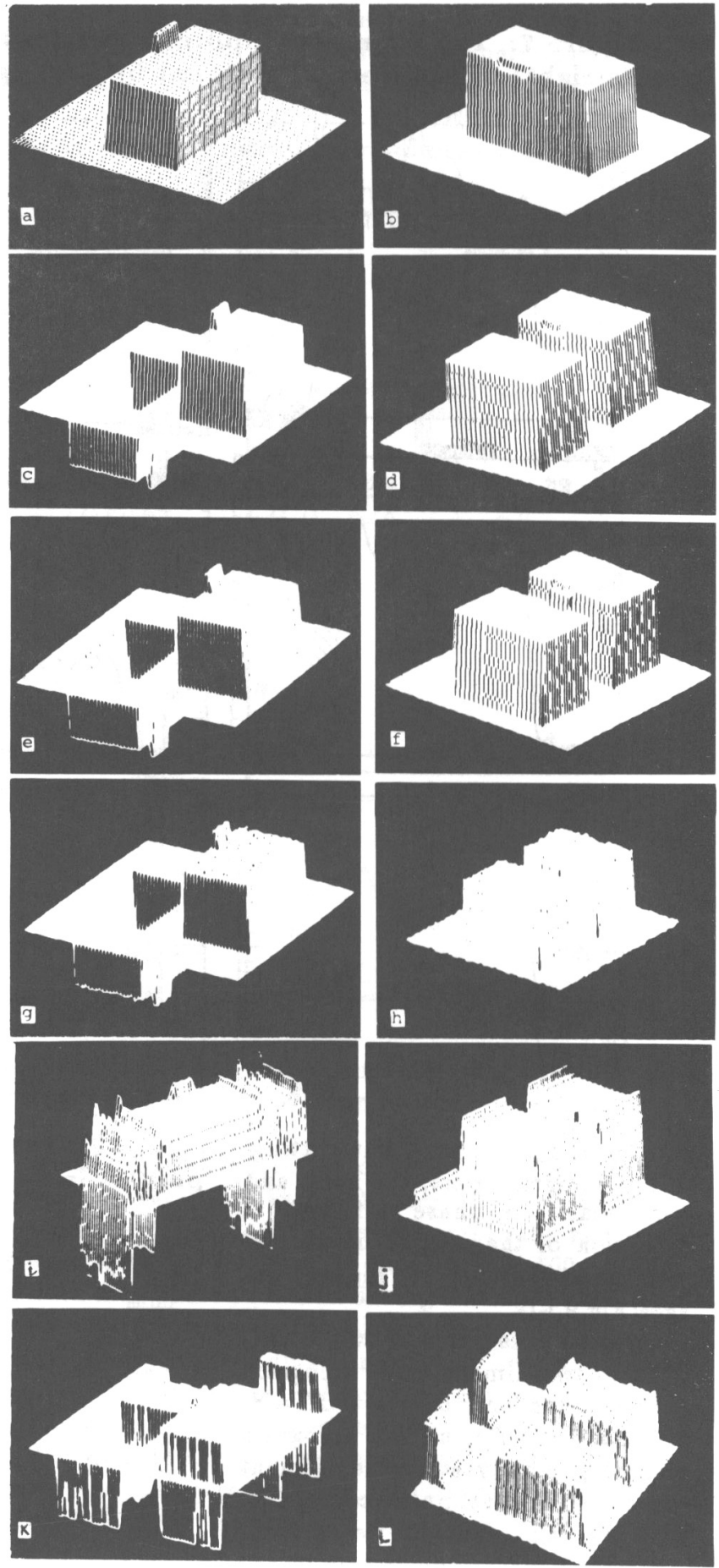
Figure 10:

The scans with numbers 1,4, C, 10, 11, 17, 18, 20 of 20 scans hologram recorded in the real experiment at the RATAN-600

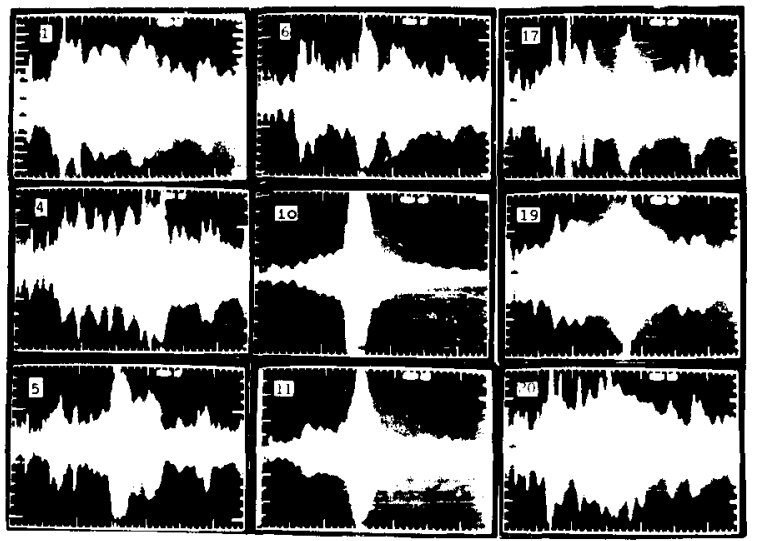

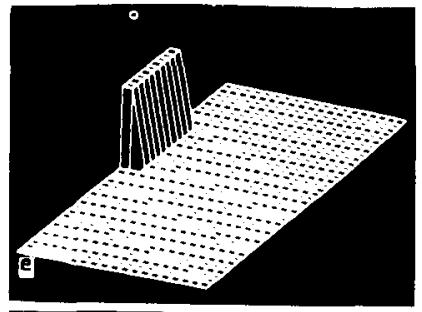
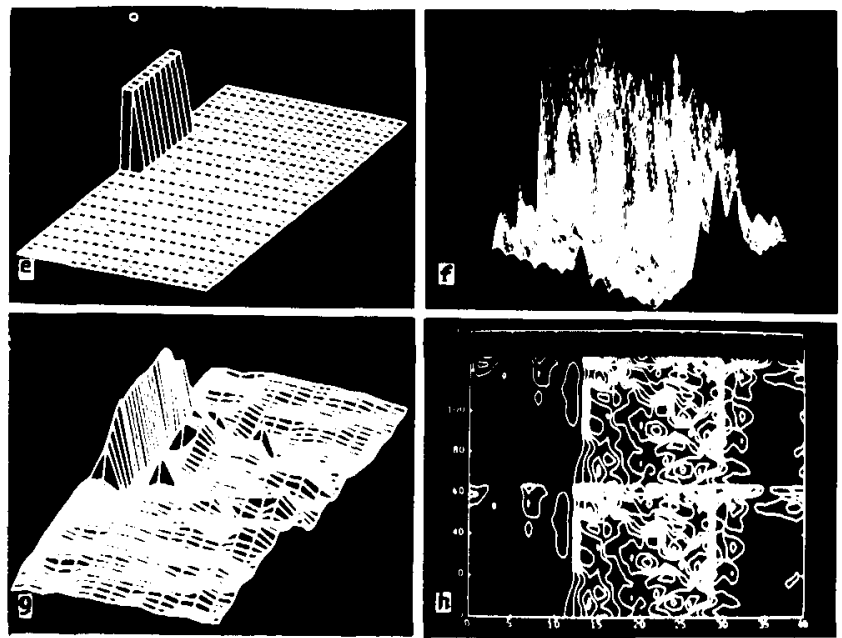

Figure 11:

The restored aperture fleld phase and amplitude of a single panel in the real experiment, $B$ - phase, f - amplitude, e - primarily installed test plate at the element surface, $h$ - aperture amplitude map.

\section{THE RATAN-600 BEAM PATTERN AT 3.2 MM}

Fig. 12 demonstrates the RATAN-600 beam pattern obtained at $3.2 \mathrm{~mm}$ and its dynamics due to antenna temperature deformations in the most anfavorable time the next day after the antenna correction. The estimation of the twodimensional RATAN-600 beam pattern at $3.2 \mathrm{~mm}$ shows that immediately after the antenna correction about $25 \%$ of energy is concentrated in the beam pattern main lobe and a holography antenna correction is desirable before the radio 
astronomical observations at $M M$ waves.

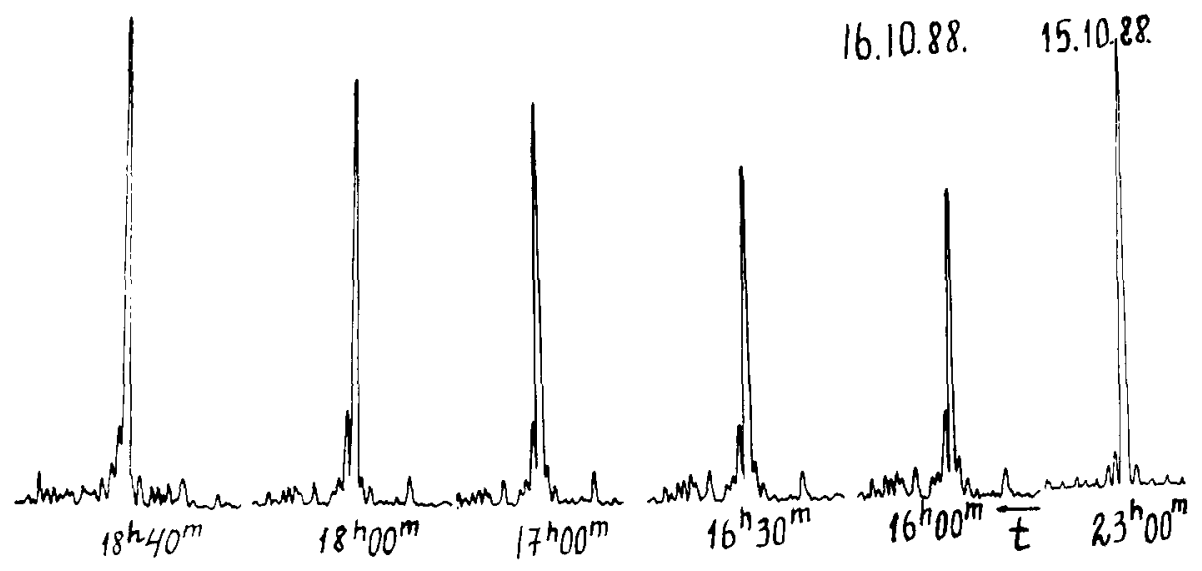

Figure 12: The RATAN-600 beam pattern at $3.2 \mathrm{~mm}$ and its dynamics in the daytime

\section{CONCLUSION}

Active holography techniques developed at the RATAN-600 can be successfully atilized at other radio telescopes. We offer cooperation to all those who need active and precise testing of a radio telescope au riace.

\section{REF EREN CES}

Bennet, J.C., Anderson, A.P., Mcinnes, P.A. and Whitaker, A.J.T. 1976, AP-24, N.3, pp.295.303.

Dvoyan, G., Pironmian, H. and Khaikin, V. 1891, Proceedinga of International Workshop on Holography in N.Arkhyz, Russia, pp.88-89.

Khaikin, V. 1981, Proceedings of XIV ESA Workshop on Antenna Measurements in Noordwijk, Netherlands.

Khaikin, V., Yaremenko, A. 1891, Proceedings of International Workshop on Hologrephy in N.Arkhyz, Russia, pp.82-84.

Korolkov, D.V., Parijskij, Yu.N. 1979, Sky and Telescope, N.4, pp.324-329.

Parijakij, Yu.N, Pinchuk, G.A., Verkhodanov, O.V.. Khaikin, V.B., Zverev, Yu.K., Zhekanis, G.V. 1992, These Proceeding8.

Romanov, G, Trehovitskij, O., Khaikin, V. and Pazakov, A. 1991, Proceedings of International Workehop on Holography in N.Arkhyz, Russia, pp.85-87. 